\title{
OBSERVAÇÕES SOBRE A LEISHMANIOSE TEGUMENTAR, CINCO ANOS APOS PROFILAXIA
}

\author{
Lais Clark Lima * \\ Mauro Célio de Almeida Marzochi** \\ Paulo Chagasteles Sobroza*** \\ Miguel Alves de Souza**
}

\begin{abstract}
LIMA, L. C. et al. Observações sobre a leishmaniose tegumentar, cinco anos após profilaxia. Rev, Saúde públ., S. Paulo, 22:73-7, 1988.

RESUMO: Em 1974 foram diagnosticados vários casos de leishmaniose tegumentar americana, no Município do Rio de Janeiro, RJ (Brasil). Naquela ocasião, foram usadas como medidas profiláticas o tratamento dos doentes e a dedetização dos domicílios e anexos, resultando na interrupção da transmissão. Com o aparecimento de novos casos, a partir de 1979, foram reestudadas algumas características de uma das localidades (Camorim) do mesmo município. De junho de 1979 a agosto de 1981, foram comprovados nessa localidade 19 casos novos de leishmaniose tegumentar americana (18 humanos e um em cão). Coletaram-se 1.149 flebótomos ( $11,4 \%$ fêmeas e $88,6 \%$ machos) com predomínio de Lutzomyia intermedia ( $95 \%)$. De acordo com o estudo dos casos, pressupóe-se que a transmissão tenha ocorrido no domicílio ou peridomicílio. Acredita-se, pois, que na área estudada, as medidas de controle, adotadas em 1974, foram eficientes por um período de aproximadamente quatro anos.
\end{abstract}

UNITERMOS: Leishmaniose mucocutânea, incidência. Lutzomyia intermedia. Ecologia de vetores.

Na cidade do Rio de Janeiro, a ocorrência de leishmaniose tegumentar americana (LTA) é conhecida desde 1915 (D'Utra e Silva ${ }^{4}$, 1915). Em 1921 ocorreram 50 casos de LTA no bairro residencial de Laranjeiras (Aragão², 1927). Nery-Guimarães e Bustamante (1954) registraram um surto epidêmico em Magé, localidade da Baixada Fluminense, próxima à cidade do Rio de Janeiro em 1947. Cinco anos depois esta epidemia havia sido pratimente extinta. De acordo com esses autores, haviam sido feitas, na área, aspersōes domiciliares com DDT para combater a malária, - que os levou a sugerir que o DDT tem ação flebotomicida e repelente para a Lutzomyia intermedia. Esta espécie de flebotomídeo, transmissora da LTA, demonstrada por Aragão ${ }^{1}$ (1922), foi encontrada naturalmente infectada por Leishmania brasiliensis (Rangel e col. $\left.{ }^{10}, 1984\right)$, é pré-adaptada ao ambiente rural $\left(\right.$ Lima $\left.^{8}, 1986\right)$ e de alta habilidade endo- fílica (Gomes e col. $\left.{ }^{7}, 1986\right)$. Devido aos seus hábitos, Forattini e col. ${ }^{6}$ (1976) admitem que a aplicação de inseticidas residuais nas habitações $e$ anexos é indicada para interromper a transmissão da LTA.

Em 1974 foram diagnosticados vários casos de LTA no bairro de Jacarepaguá, Rio de Janeiro, RJ (localidades: Camorim, Sacarrão e Cabungui). $\mathrm{Na}$ ocasião, foram adotadas como medidas profiláticas, pelo "grupo de trabalho coordenador das atividades de estudo e controle da leishmaniose tegumentar americana", na área de atuação do Posto de Saúde Samuel Libânio (Jacarepaguá), o tratamento dos doentes e a dedetização dos domicílios e anexos peridomiciliares. Esta foi feita pela Superintendência de Campanha de Saúde Pública (SUCAM), em 27 de junho de $1974^{* * * *}$, o que levou à interrupção da transmissão (Sabroza ${ }^{12}$, 1981). A constatação de novos casos no mes-

* Laboratório de Leishmaniose do Centro de Pesquisas René Rachou da Fundação Oswaldo Cruz (FIOCRUZ) - Caixa Postal 1743 - 30190 - Belo Horizonte, MG - Brasil.

Departamento de Ciências Biológicas da Escola Nacional de Saúde Pública - FIOCRUZ - Caixa Postal 926 - 21040 - Rio de Janeiro, RJ - Brasil.

*: Departamento de Epidemiologia da Escola Nacional de Saúde Pública - FIOCRUZ - Caixa Postal 926 - 21040 - Rio de Janeiro, RJ - Brasil.

:**:: De acordo com dados de relatório da campanha de controle de leishmaniose em Jacarepaguá, Rio de Janeiro - Doc. interno. 
mo bairro, a partir de 1979, levou-nos a reestudar algumas características de uma das localidades da região.

\section{MATERIAL E MÉTODOS}

\section{Ârea de Estudo}

O local escolhido foi a localidade de Camorim, por ter maior número de domicílios concentrados, por estar mais próximo de centros industriais, comerciais e urbanos dos bairros de Jacarepaguá e Barra da Tijuca, por ser de fácil acesso, por ter apresentado alta taxa de incidência de LTA - 6,2\%, no ano de 1974 (Sabroza $^{12}$, 1981) e por haver informações sobre os flebotomíneos lá existentes durante este ano.

A área estudada (Camorim) situa-se na vertente atlântica do Maciço da Pedra Branca, no bairro de Jacarepaguá, Rio de Janeiro, RJ, a $43^{\circ} 25^{\prime} \mathrm{W}$ e $22^{\circ} 55^{\prime} \mathrm{S}$. Trata-se de localização periurbana, com afloramentos graníticos e gnaíssicos (Fig.), consistindo, de acordo com Sabroza' ${ }^{12}$ (1981), em um anfiteatro formado por várzea de natureza sedimentar argilo-arenosa, limitada na abertura pela Estrada dos Bandeirantes e lateralmente pelas Pedras Rosilha e Negra, cortada pelos rios Camorim e Caçambê. As encostas têm as cotas mais altas, em torno de $500 \mathrm{~m}$.

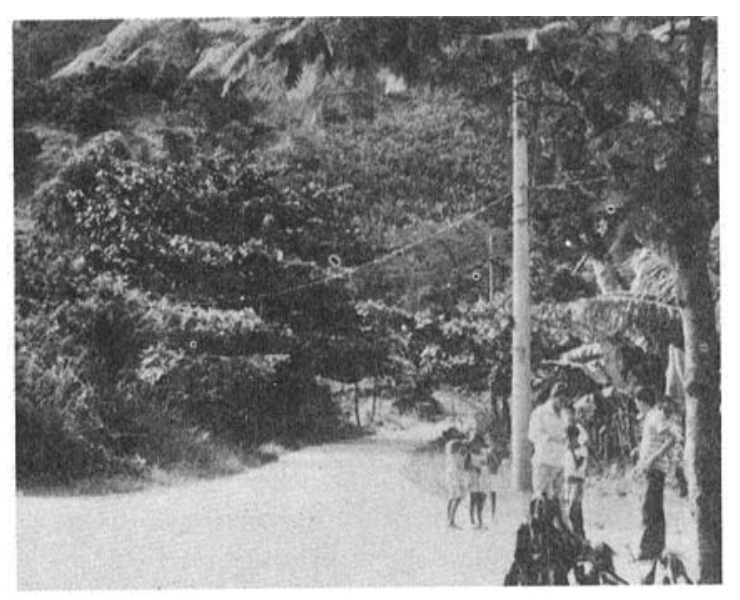

Fig. Aspecto da localidade do Camorim, mostrando o tipo de arruamento, a cobertura vegetal com plantação de bananeiras e afloramento rochoso.

No Camorim, a floresta tropical começou a ser derrubada para fornecer lenha aos engenhos de açúcar; posteriormente floresceu nas encostas a plantação de cafezais (nos séculos XVI e XIX), laranjais (a partir de 1930) e bananais (Secretaria Municipal de Planejamento $\left.^{13}, 1979\right)$. Atualmente, parte das terras foi ocupada por capoeiras e capinzais, sendo encontrados, nas vertentes das reservas florestais (limitadas aos espigões mais elevados), bananais, árvores frutíferas, havendo ainda árvores ornamentais. A baixada adquire características urbanizadas com arruamento e de favelas (Fig.). Na região, uma área de $26 \mathrm{~km}^{2}$, considerada área preservada da União, era uma antiga floresta protetora de mananciais d'água. Vale ressaltar que de 1972 a 1978 foram constatados desmatamentos nas encostas da Pedra Negra e Serra do Nogueira, voltadas para as estradas do Camorim e Bandeirantes, atingindo esta última a cota dos $500 \mathrm{~m}$ (Secretaria Municipal de Planejamento $\left.{ }^{13}, 1979\right)$. A cobertura vegetal, entretanto, é suficiente para abrigar aves, pequenos roedores, marsupiais, sagüis, sendo por vezes encontrados outros mamíferos de pequeno porte.

O clima regional, segundo a classificação de Köeppen, é do tipo AW, caracterizado por um verão úmido e chuvoso e um inverno seco. A temperatura média das máximas é de $29,7^{\circ} \mathrm{C}$ e a média das mínimas de $20,7^{\circ} \mathrm{C}$. A precipitação anual é de $1.269,4 \mathrm{~mm}$ (Secretaria Municipal de Planejamento ${ }^{14}, 1980$ ).

Quanto às condições de saneamento, a localidade é servida por água proveniente de mananciais ou poços; não há sistema de esgotos, os dejetos são depositados em fossas ou levados por valas aos rios próximos.

Quanto ao tipo de material de construção e iluminação, na várzea predominam casas de alvenaria e com iluminação elétrica, sendo as restantes de madeira ou estuque e sem eletricidade. $\mathrm{Na}$ encosta, algumas casas são de alvenaria, mas não há iluminação elétrica.

Das 223 construções por nós percorridas no local, 18 têm outras finalidades que não a de moradia, seis estão desabitadas, 23 são sítios e casas de campo para recreação, e as 176 restantes são moradias do pessoal local (antigos posseiros, funcionários públicos da reserva florestal, caseiros, agricultores e operários).

\section{Procura de Casos Autóctones de LTA}

Com o objetivo de verificar a presença de leishmaniose tegumentar na área, fazia-se a busca de casos através da visitação às residências, divulgando-se a existência da doença no local e interrogando-se os moradores sobre a presença de lesão em atividade ou problema de pele ou mucosa.

Os casos suspeitos eram encaminhados ao Posto de Saúde Samuel Libânio, ocasião em que era preenchida uma ficha clínico-epidemiológica com dados tais como sexo, grupo 
etário, tipo e local de ocupação, local e tempo da lesão, tempo de residência, estado migratório e outros.

Os casos só eram considerados confirmados quando, após o exame clínico e constatação da presença de lesão suspeita, eram comprovados através da intradermorreação de Montenegro e/ou por pesquisa de leishmania na lesão.

\section{Capturas de Flebotomíneos}

As capturas de flebotomíneos foram realizadas de abril de 1980 a maio de 1981 , em domicílios previamente selecionados, com isca humana e nas paredes internas e externas; e nos peridomicílios, em troncos de árvores, abrigos de porcos, galinheiros e fendas de rochas. Para tais capturas foi utilizado o capturador manual de Castro. Foram também empregados em diversos locais do peridomicílio, o papel com óleo e a armadilha de Chaniotis.

\section{RESULTADOS}

De junho de 1979 a agosto de 1981, foram comprovados 19 casos novos de LTA, sendo 18 humanos e um, em cão. Considerando-se o tempo de incubação da doença em torno de três meses, de acordo com as informações obtidas sobre o tempo de aparecimento das lesões, a primeira infecção ocorreu em janeiro de 1979. Não houve diferença na frequiência de casos humanos com relação ao sexo (9:9). Quanto à idade, ocorreram casos em adultos, adolescentes e crianças, sendo a maior freqiiência (8) naqueles com até 4 anos de idade. O maior número de casos (12) foi observado entre os moradores da encosta.

Foram realizadas 97 horas de capturas de flebotomíneos. De acordo com a Tabela, em 64 horas de capturas positivas, coletaram-se 1.149 flebótomos. Entre eles, 131 fêmeas $(11,4 \%)$ e 1.018 machos $(88,6 \%)$. Houve um predomínio de $L$. intermedia: 1.092 (95\%) exemplares, seguida de L. migonei: $\mathbf{5 2}$ $(4,5 \%)$; L. pelloni: $3(0,3 \%)$, um exemplar de Lutzomyia schreiberi e um de Lutzomyia firmatoi.

As capturas foram mais eficientes com isca humana, em abrigos de porcos e troncos de árvores, nessa ordem, tendo sido pouco produtivas nos galinheiros (Tabela). $\mathrm{O}$ rendimento obtido com o capturador de Castro foi maior do que com os métodos do papel com óleo e armadilha de Chaniotis. Com isca humana, no interior do domicílio, capturou-se maior número de $L$. migonei, predominando machos. Nas fendas de rochas, as capturas foram negativas.

\section{DISCUSSÃO}

As características levantadas sobre a ocorrência de LTA, no Camorim, durante o período deste estudo, foram as mesmas do surto de 1974, estudado por Sabroza ${ }^{12}$ (1981).

As pessoas são atingidas pela enfermidade, independentemente de sexo, ocupação ou tipo de habitação. Vale salientar que a maior incidência ocorreu em crianças. A transmissão, portanto, devia ocorrer no peridomicílio ou no interior das casas.

As três espécies de flebótomos quantitativamente mais representativas nas capturas em questão, $L$. intermedia, $L$. migonei e $L$. pello$n i$, nessa ordem, foram as mesmas que ocorreram em 1974, sendo que de julho (após a borrifação) a dezembro de 1974 , as capturas do Camorim foram negativas, enquanto na estação controle (não dedetizada) as capturas foram positivas (Ministério da Saúde*, 1974).

Barreto $^{3}$ (1943) verificou que, em geral, nas capturas noturnas, há uma predominância de machos sobre as fêmeas, fato também verificado em 1974 e no presente estudo.

Salientamos que a $L$. migonei, de hábitos mais silvestres, embora com elevada antropofilia (Barreto , 1943; Forattini ${ }^{5}, 1954$ e Rangel e col. ${ }^{11}$, 1986), foi aqui capturada, em maior densidade, dentro do domicílio, fato este também observado, no mesmo bairro**, com isca humana, embora nesta captura tenham predominado machos.

Segundo Sabroza ${ }^{12}$ (1981), no Camorim "não foi registrado nenhum caso que tivesse iniciado a doença entre setembro de 1974 e dezembro de 1978, enquanto que nas áreas vizinhas, Pau de Fome e Guaratiba, que não foram trabalhadas, a transmissão ocorreu com intensidade". Assim sendo, após levantamento feito no Posto de Saúde Samuel Libânio e a análise das fichas clínico-epidemiológicas dos pacientes deste estudo, acreditamos que, na área em questão, as medidas de controle, adotadas em 1974, foram eficazes durante um período de aproximadamente quatro anos.

* De acordo com dados de relatório da campanha de controle de leishmaniose em Jacarepaguá, Rio de Janeiro - Doc. interno.

Dados não publicados; comunicação pessoal de M. Alves de Souza (Escola Nacional de Saúde Pública - FIOCRUZ). 


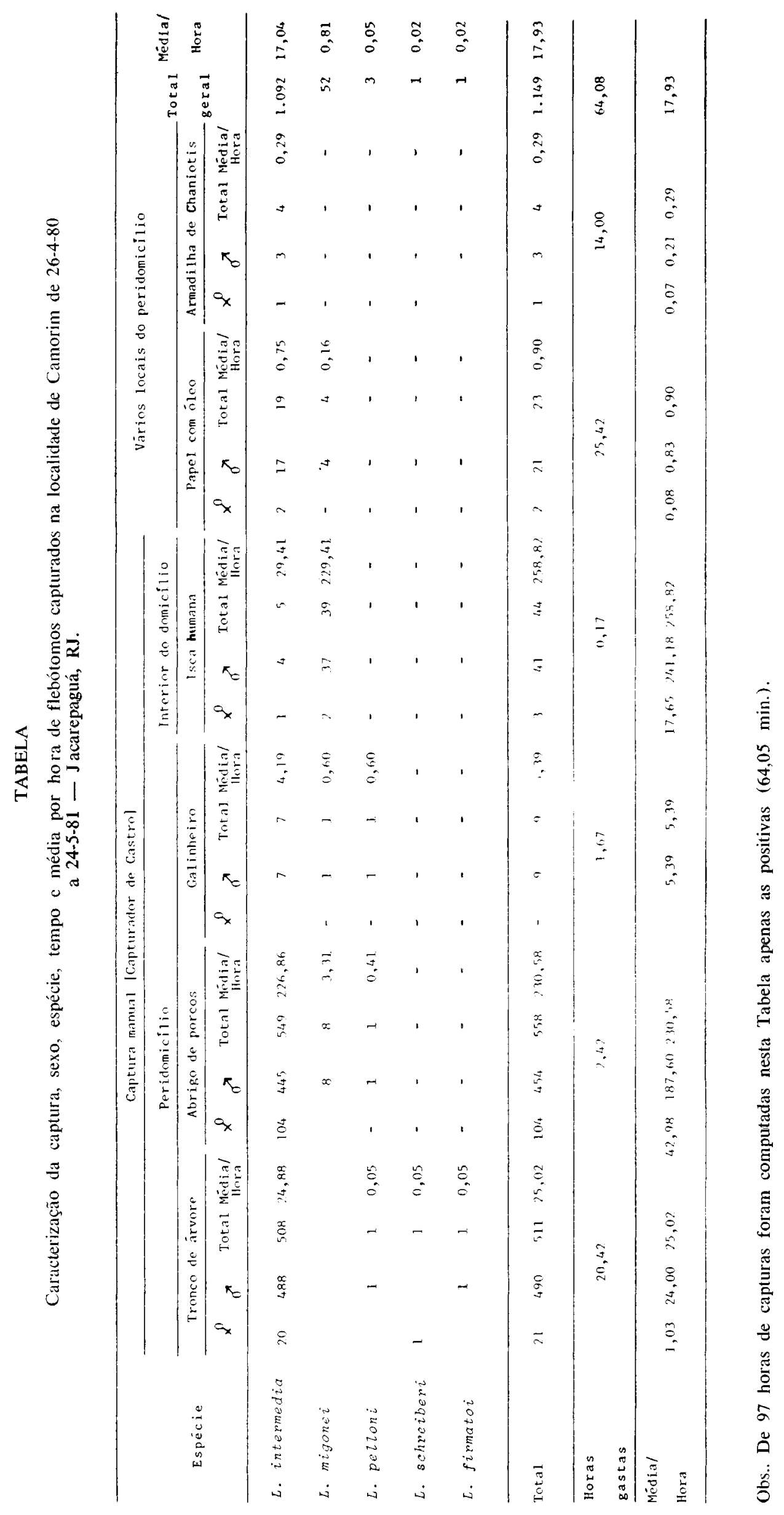


LIMA, L. C. et al. [Observations of cutaneous leishmaniasis in Brazil, after five years of prophylaxis]. Rev. Saúde públ., S. Paulo, 22:73-7, 1988.

ABSTRACT: In 1974 several cases of cutaneous leishmaniasis were diagnosed in Jacarepaguá, Rio de Janeiro, RJ (Brazil). At that time the patients were treated, the houses and surroundings were sprayed with DDT, and this resulted in the interruption of transmission. The emergence of new cases in 1979 led to a resumption to the study of some characteristics of one of the localities (Camorim) in this area. Eighteen new human cases of cutaneous leishmaniasis and one in a dog were confirmed between June 1979 and August 1981. One thousand hundred and forty nine sand flies were collected (11.4\% females and $88.6 \%$ males) with predominance of Lutzomyia intermedia (95\%). The study of the human cases led to the supposition that the transmission of the disease may take place in the domiciliary or peridomiciliary environment. It is believed that, at least for the studied area, the control measures taken in 1974 were efficient for four years.

UNITERMS: Leishmaniasis, visceral, occur. Lutzomyia intermedia. Ecology, vectors.

\section{REFERENCIAS BIBLIOGRÄFICAS}

1. ARAGÃO, H. B. Transmissão da leishmaniose no Brasil, pelo Phlebotomus intermedius. Brasil méd., Rio de Janeiro, 36:129-30, 1922.

2. ARAGÃO, H. B. Leishmaniose tegumentar e sua transmissão pelos phlebótomos $\mathrm{Mem}$. Inst. Oswaldo Cruz, 20:177-87, 1927.

3. BARRETTO, M. P. Observações sobre a biologia em condições naturais, dos flebótomos do Estado de São Paulo (Diptera, Psychodidae). São Paulo, 1943. [Tese de Livre Docência - Faculdade de Medicina da USP].

4. D'UTRA e SILVA, O. Sobre a leishmaniose tegumentar e seu tratamento. Mem. Inst. Oswaldo Cruz, 7:213-48, 1915.

5. FORATTINI, O. P. Algumas observações sobre a biologia dos flebótomos (Diptera, Psychodidae) em região da bacia do Rio Paraná (Brasil). Arq. Fac. Hig. S. Paulo, 8:15-136, 1954.

6. FORATTINI, O. P. et al. Observações sobre a transmissão da leishmaniose tegumentar no Estado de São Paulo, Brasil. Rev. Saúde públ., S. Paulo, 10:31-43, 1976.

7. GOMES, A. C. et al. Ecological aspects of american cutaneous leishmaniasis. 4 - Observations on the endophilic behavior of the sandfly and the vectorial role of Psychodopygus intermedius in the Ribeira Valley region of S. Paulo State, Brasil. Rev. Satide públ., S. Paulo, 20:280-7, 1986.

8. LIMA, L. C. Ruralização da Lutzomyia intermedia um provável caso de pré-adaptação. Rev. Salide públ., S. Paulo, 20:102-4, 1986.
9. NERY-GUIMARÃES, F. \& BUSTAMENTE, F. M. A aplicação domiciliar de DDT como base de profilaxia das leishmanioses: estudo de um foco de leishmaniose muco-cutânea cinco anos depois da aspersão periódica com aquele inseticida. Rev. bras. Malar., 6:127-30, 1954.

10. RANGEL, E. F. et al. Infecção natural de Lutzomyia intermedia Lutz \& Neiva, 1912, em área endêmica de leishmaniose tegumentar no Estado do Rio de Janeiro. Mem. Inst. Oswaldo Cruz, 79:395-6, 1984.

11. RANGEL, E. F. et al. Flebótomos de Vargem Grande, foco de leishmaniose tegumentar no Estado do Rio de Janeiro. Mem. Inst. Oswaldo Cruz, 81:347-9, 1986.

12. SABROZA, P. C. O domicílio como fator de risco na leishmaniose tegumentar americana. Estudo epidemiológico em Jacarepaguá, Município do Rio de Janeiro. Rio de Janeiro, 1981. [Dissertação de Mestrado - Escola Nacional de Saúde Pública, FIOCRUZ].

13. SECRETARIA MUNICIPAL DE PLANEJAMENTO. Diagnóstico do desmatamento nos maciços da Tijuca, Pedra Branca e Gericinó. Rio de Janeiro, 1979.

14. SECRETARIA MUNICIPAL DE PLANEJAMENTO. Informações básicas da cidade do Rio de Janeiro. Rio de Janeiro, 1980.

Recebido para publicação em: 16/3/1987 Reapresentado em: 13/11/1987

Aprovado para publicação em: 16/11/1987 